\title{
Effect of Time Delay on Flying Qualities: An Update
}

\section{Rogers E. Smith and Shahan K. Sarrafian}

\section{FOR REFERENCF:}

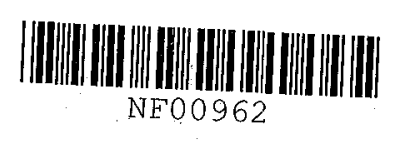

\section{N/SN}

National Aeronautics and Space Administration

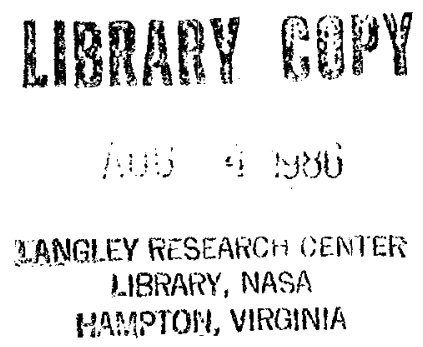

HAMPTOM, VIRGINIA 


\section{Effect of Time Delay on Flying Qualities: An Update}

Rogers E. Smith and Shahan K. Sarrafian

Ames Research Center, Dryden Flight Research Facility, Edwards, California

\section{N/SA}

National Aeronautics and

Space administration

\section{Ames Research Center}

Dryden Flight Research Facility

Edwards, California 93523-5000

$$
186-28092 \#
$$




\title{
EFFECT OF TIME DELAY ON FLYING QUALITIES: AN UPDATE
}

\author{
Rogers E. Sinith* and Shahan K. Sarrafiant \\ NASA Ames Research Center \\ Dryden Flight Research Facility \\ Edwards, California
}

\begin{abstract}
Flying qualities problems of modern, fullauthority electronic flight control systems are most of ten related to the introduction of additional time delay in aircraft response to a pilot input. These delays can have a significant effect on the flying qualities of the aircraft. This paper reexamines time delay effects in light of recent flight test experience with aircraft incorporating new technology. Data from the $X-29 A$ forward-swept-wing demonstrator, a related preliminary in-flight experiment, and other flight observations are presented. These data suggest that the present MIL-F-8785C allowable-control system time delay specifications are inadequate or, at least, incomplete. Allowable time delay appears to be a function of the shape of the aircraft response following the initial delay. The cockpit feel system is discussed as a dynamic element in the flight control system. Data presented indicate that the time delay associated with a significant low-frequency feel system does not result in the predicted degradation in aircraft flying qualities. The impact of the feel system is discussed from two viewpoints: as a filter in the control system which can alter the initial response shape and, therefore, the allowable time delay, and as a unique dynamic element whose delay contribution can potentially be discounted by special pilot loop closures.

\section{Nomenclature}

FAS roll stick force input, ib

$K_{1}$ pilot model feedback gain

KFS feel system gain, in/lb

Kp lateral command gain, deg/sec/in

LATHOS lateral higher order systems

p roll rate, $\mathrm{deg} / \mathrm{sec}$

Plo pilot-induced oscillation

PR pilot rating

$5 \quad$ Laplace operator

$\mathrm{T}_{1} \quad$ pilot model time constant, sec
\end{abstract}

*Aerospace Research Pilot. Member AIAA. †Aerospace Engineer. Member AIAA.

$\begin{array}{ll}\delta_{A} & \text { aileron position, deg } \\ \delta_{A S} & \text { roll stick position, in } \\ \zeta_{\mathrm{FS}} & \text { feel system damping ratio } \\ \zeta_{n} & \text { neuromuscular damping ratio } \\ \tau_{\mathrm{e}} & \text { equivalent time delay, sec } \\ { }^{\tau_{\mathrm{eff}}} & \text { effective time delay, sec } \\ { }^{\tau_{R}} & \text { roll mode time constant, sec } \\ { }^{\omega_{\mathrm{FS}}} & \text { feel system natural frequency, rad/sec } \\ { }^{\omega_{n}} & \text { neuromuscular natural frequency, } \mathrm{rad} / \mathrm{sec}\end{array}$

\section{Introduction}

The advent of modern, full-authority electronic flight control systems produced many exciting advances in aircraft handling and performance capabilities. Unfortunately, this improved capability has not evolved without cost. Chief amony the problems related to this modern technology is the introduction of additional time delay in the response of the aircraft to pilot input. These time delays can produce a significant deyradation in the flying qualities of the aircraft during demanding tasks.

This paper examines time delay effects in light of recent flight test experience with aircraft such as the $X-29 A$ forward-swept-winy demonstrator which incorporate new technology. Early examples of aircraft with high-authority electronic flight control systems (such as the F-18A, Tornado, YF-17, and the space shuttle) had specific flying qualities problems related to time delay exposed during their development proyrams. As a result, new requirements on allowable flight control system time delay were included in the latest military flying qualities specification, MIL-F-8785C. 1 Recent experience with the $X-29 \mathrm{~A}$ aircraft and later versions of the $F-18 \mathrm{~A}$ aircraft raised some questions about the validity or, at least, completeness of these specifications on allowable control system time delay.

The general purpose of this paper is to present questions related to allowable time delay which have recently surfaced during the $x-29 A$ flight tests and a related preliminary flying qualities research experiment. The specific purposes are to: 
1. Present a summary of the pertinent MIL-F-8785C specifications, and review the fundamental background information and definitions related to flight control system time delay.

2. Present new data and flight test observations which bring the existing MIL-F-8785C time delay specifications into question. These data indicate that the effect of a given overall control system time delay is a function of the distribution and character of the control system elements. There is evidence from these data and other sources that the allowable control system time delay may be a function of the shape of the initial aircraft response after the time delay.

3. Address, specifically, the role of the cockpit feel system, which relates the pilot's input force to the control stick displacement in the overall flight control system. The feel system is a potentially unique dynamic element; the pilot has direct access to both the input force and the output stick displacement. The question to be addressed is whether the feel system is merely another filter in the overall control system, or whether it is a unique dynamic element whose time delay contribution can be either ignored or reduced by the pilot.

This paper is based on data and observations yathered from the perspective of the pilot and fliyht test engineer. Although the data base for the paper may be somewhat limited, the basic themes in the paper are supported by flight observations. The authors hope the fundamental flight observations presented herein will encourage the evolution of a larger data base with which analysts can provide additional insight.

\section{Background Information}

Because the primary purpose of this paper is to present some new insights on the subject of time delay and flying qualities, a complete review of the background data is beyond our scope. However, as a foundation for the discussion in this paper, a brief and basic review of the time delay definitions and the important effects of time delay on flying qualities is presented. A more complete discussion of the subject can be found in Ret. 2 .

Iime Delay.

To a pilot, time delay is the dead time between his force input to the stick and the beginning of any aircraft response or output. This delay can come from a variety of sources within the flight control system.

A systell that reproduces the exact shape of an input after an interval of dead time is defined as exhibitiny transport, or pure, time delay. In modern diyital flight control applications, this pure time delay is introduced by the digital implementation of the control laws. However, the majority of time delay in modern electronic flight control applications is not caused by these pure digital time delays. Typically, the complexity of modern control system design strategies results in cascading numerous dynamic elements which can introduce a perceived delay in the initial response of the aircraft to a pilot input. This form of time delay is often referred to as "equivalent" or "effective" time delay, depending on the measurement method. Each method represents an approximation of the dead time sensed by the pilot.

\section{Time Delay Measurement}

"Equivalent" time delay in a flight control system is measured using frequency domain techniques. It can be "measured" by matching the frequency response of the complex high-order system over a specific frequency range with a familiar low-order model, which includes a pure time delay term. 3 Typically, the pitch rate or roll rate transfer functions are analyzed.

"Effective" time delay in a flight control system is measured using time domain techniques. It is measured as the difference between the time of application of a step input and the intersection of the maximum slope tangent to the response, as shown in Fig. 1. The effective time delay measure 4 does not require an assumed low-order model. Again, pitch rate or roll rate responses to step inputs are typically used for this purpose.

It is important that time delay flying qualities data, design guidelines, or specifications include a clear definition of the required measurement technique involved. The frequency and time domain measurement techniques do not always produce exactly the same answers. For the remainder of this paper, the general term "time delay" is used unless data involving a specific measurement technique are involved.

Time Delay and the Task

The evaluation of highly augmented aircraft with appreciable time delay is very much a function of pilot technique and the degree of precision demanded by the task. For example, the flying qualities of an aircraft with significant time delay may be satisfactory for the approach phase of the landing task but deteriorate significantly near touchdown as the required task precision increases.

Results of the NASA F -8 research program 5 shown in Fig. 2, illustrate that the task performance demands are an important flying qualities factor. For the precision, or high-stress pitch landing task, which included a lateral of $f$ set maneuver and a specific touchdown zone, the degradation in pilot rating is much steeper than for the low-stress task. An alternate description of the task differences for these examples would be to say that the precision or high-stress task requires a higher inner-loop bandwidth than the low-stress, straight-in approach. 
Also shown in Fig. 2 are data for the NT-33 airplane ${ }^{6}$ for a similar task which correlates well with the F-8 high-stress data. The lowstress task involved a straight-in approach with no touchdown zone constraints; the data trends are similar to those obtained in a sophisticated fixecl-base simulator, 7 using the task and configurations from the NT-33 program reported in Ref. 6.

The preceding brief review establishes that control system time delay is a critical flying qualities factor. Careful attention must be yiven to measurement technique, task details, and pilot technique during flight test or flying qualities evaluations.

Before the discussion of new data and flight observations that bring into question the present allowable control system time delay specifications, a review of present specifications is in order.

\section{MIL-F-8785C Time Delay Specifications}

The MIL-F-878bC flying qualities specifications on allowable time delay address the dynamic characteristics of the primary flight control system which includes pitch, roll, and yaw stability augmentation systems, and all associated mechanisms and devices. In addition, aircraft response to cockpit control deflection and control force is to be smooth and linear for all control input amplitudes. This aircraft response to cockpit control force must not exhibit a time delay longer than the following for a pilot-initiated step control force input:

$$
\begin{aligned}
& \text { Level 1.... } 0.10 \mathrm{sec} \\
& \text { Level 2.... } 0.20 \mathrm{sec} \\
& \text { Level } 3 \text {. . . } 0.25 \mathrm{sec}
\end{aligned}
$$

These allowable time delay requirements are also applicable to values of equivalent time delay derived from an equivalent system frequency domain match of the aircraft response to cockpit controls. As previously stated, this delay refers to the pure time delay term in the resulting loworder model. These delay requirements cover all aircraft and missions. One obvious weakness in the present time delay requirements is that precise definitions of the required time delay measurement techniques are not given. Another area of concern is that the allowable time delay values are not a function of any other factors that affect the shape of the response after the delay time. For example, the data in Ref. 4 showed that the allowable lateral time delay was a function of the value of the roll mode time constant. Also note that the time delay requirements are based on stick force inputs. For aircraft that use stick displacement as a command, the delay of the feel system is therefore included in the overall delay for comparison with the MIL-F-8785C time delay thresholds.
Recent Time Delay Flying Qualities Data/0bservations

$X-29 A$ Flying Qualities Evaluations

The $\mathrm{X}-29 \mathrm{~A}$ primary flight control system is a relatively complex digital design 8 which uses stick position for the command signal to the computers. Thus, the feel system is in series in the command path of the flight control system. Recent observations during the $X-29 A$ advanced technology demonstrator flight test program raised questions about the applicability of current MIL-F-8785C allowable time delay requirements.

For example, frequency domain equivalent system analysis referenced to stick force, as required by MIL-F-8785C, showed the X-29A to have unacceptably high values of equivalent time delay (Level 3) at a number of flight conditions. Initial flight evaluations using realistic precision formation tasks indicate that the $X-29 A$ is typically borderline Level 1/Level 2 for these tasks; for the precision roll tasks it is a solid Level 1 aircraft. One unique feature of the $X-29 A$ flight control system is a relatively "slow" feel system. In the roll axis, the feel system can be characterized as follows:

$$
\frac{\delta A S}{F_{A S}}=\frac{K F S}{s^{2}+2(0.7)(13) s+(13)^{2}}
$$

This lateral feel system, which has a spring gradient of $2 \mathrm{lb} / \mathrm{in}$, contributes approximately $0.10 \mathrm{sec}$ of equivalent time delay. For the $x-29 \mathrm{~A}$ this slow feel system is responsible for approximately 45 percent of the overall time delay which, according to MIL-F-8785C, should yield Level 3 flying qualities.

When the feel system dynamics are excluded from the equivalent system analysis procedure, the resulting equivalent delay values fall in the MIL-F-8785C Level 1/Level 2 region, which is then consistent with the flight evaluations. The preliminary flying qualities results from the $X-29 A$ tests indicate no pilot performance degradation due to time delay. These results are significantly different than the flying qualities levels predicted by the MIL-F-8785C allowable time delay requirements.

The $x-29 A$ results show that referencing the time delay measurements to stick position provides good correlation between the flight results and the MIL-F $-8785 \mathrm{C}$ requirements. However, if the time delay measurements are referenced to stick force, the present MIL-F-8785C allowable time delay requirements are not apparently applicable to the $X-29 A$ case. As discussed, the reason for this anomaly may be related to the $X-29 A$ feel system characteristics. The role of the feel system in the pilot/aircraft combination is the central issue in a separate flying qualities experiment presented later in this paper. 


\section{F/A-18 Control Law Development}

The evolution of the $F / A-18$ flight control system during the flight test program resulted in numerous changes that improved the flying qualities of the aircraft, particularly in the area of time delay. ${ }^{9}$ Initially, the flight control system was commanded by stick force. The necessary forward path filtering, in addition to other structural filtering requirenents, resulted in unsatisfactory levels of equivalent time delay according to MIL-F-8785C. Flying qualities evaluations of the early versions of the $F / A-18$ did, in fact, expose time-delay-related problems.

Subsequent revisions to the control laws in the flight control system included a change to position command from the original force command design. As a result of the extensive revision to the control system, including use of position cominand, equivalent time delays were reduced by 50 percent. Further, the equivalent time delays measured from stick position, which excluded feel system dynamics, moved into the Level 1 range in MIL-F-8785C. The overall assessment of flying qualities of the F/A-18 correlates with this method of excluding the delay from the feel system.

Although the F/A-1.8 feel system is "fast" coinpared with that of the $X-29 A$ (the feel system natural frequency is approximately double the $X-29 A$ value), the correlation of flight results with the MIL $-F-8785 C$ values is better if the feel system is excluded. Analysis within the $F / A-18$ program is typically done without including the feel system.

\section{NT-33 Aircraft Evaluation}

The observations noted on the $X-29 A$ and the $F / A-18$ prompted a cursory evaluation of feel systeln time delays on the USAF/Calspan NT-33 variable stability aircraft. This undocumented evaluation concentrated on the effect of feel system time delays on lateral flying qualities in the visual approach and landing tasks. Two basic configurations in the lateral axis were evaluated, and both had an overall system delay of between 0.20 and $0.25 \mathrm{sec}$. The first configuration included a relatively fast feel system $\left(\zeta_{F S}=0.7, \omega_{F S}=26.0\right.$ $\mathrm{rad} / \mathrm{sec}$ ) whose equivalent time delay was approxinately $0.05 \mathrm{sec}$. The remaining delay in the flight control system was downstream of the feel system in the command path. For the second configuration, a slow feel system was substituted for the fast feel system in the first configuration. This feel system contributed approximately 0.10 $\mathrm{sec}$ of equivalent time delay $\left(\zeta_{\mathrm{FS}}=0.7\right.$, wFS $=$ $13.0 \mathrm{rad} / \mathrm{sec}) ; 0.05 \mathrm{sec}$ of equivalent time delay was also removed downstream of the feel system to keep the overall time delay of the two configurations the same.

The second configuration with the slow feel system exhibited a slight tendency toward PIO but was controllable in the lateral-offset landing task. This configuration approximated an earlier version of the $X-29 A$ lateral power approach model which was simulated in the USAF/Calspan Total InFlight Simulator. The fact that the observations from both simulations were very similar adds some credibility to this informal NT-33 evaluation.

In contrast, the first configuration with the fast feel system and the same overall time delay was unflyable near the ground because of a divergent lateral PI0. The flying qualities of the two configurations in this superficial evaluation were very different, yet using the MIL-F-8785C time delay boundaries to compare, they should have been the same.

The observations and evaluations from the $X-29 A$ test program, supported by the examples from the F/A-18 program, and the informal NT-33 evaluation indicate that the present MIL-F-8785C allowable time delay requirements are potentially invalid or, at least, incomplete. These examples show that the allowable time delay is a function of the distribution and character of the flight control system time delay. In particular, there is evidence that the feel system is perhaps a unique dynamic element whose time delay contribution may be, to some degree, discounted.

The preceding observations served as a catalyst for a feel system investigation using the Calspan Learjet in-flight simulator. This investigation was very limited in scope because of economic constraints, but was intended to provide more insight into the time delay questions raised by the $X-29 A$ flight test program.

\section{Feel System Investigation}

The objective of this brief preliminary investigation was to provide additional insight into the effects of feel system dynamics on aircraft lateral handling qualities in the approach and landing task.

\section{Experiment Details}

For this experiment, two lateral feel systems were evaluated. The fast feel system, which contributed approximately $0.05 \mathrm{sec}$ of equivalent time delay is represented:

$$
\frac{\delta_{A S}}{F_{A S}}=\frac{K_{F S}}{s^{2}+2(0.6)(26) s+(26)^{2}}
$$

The slow feel system contributed about double the equivalent time delay, $0.10 \mathrm{sec}$, and is represented:

$$
\frac{\delta_{A S}}{F_{A S}}=\frac{K_{F S}}{s^{2}+2(0.6)(13) s+(13)^{2}}
$$

Two levels of overall control system equivalent time delay, measured from stick force to initial control surface response, were considered: $0.15 \mathrm{sec}$ and $0.27 \mathrm{sec}$. The general arrangement of the control system is shown in Fig. 3, with the flight control system commanded by stick position. For the evaluations of each level of overall time delay with both feel systems, appropriate incre- 
ments of transport time delay were employed to keep the overall time delay constant. The evaluation configurations are illustrated in Fig. 4. A "benchmark" configuration with minimum overall delay was included for reference.

A fixed roll mode time constant of approximately $0.30 \mathrm{sec}$ was used for all configurations and the dutch roll mode was essentially suppressed. The lateral roll rate transfer function is presented:

$$
\frac{\mu}{F_{A S}}=\frac{K F S}{s^{2}+2 r_{F S}{ }^{\omega} F S^{s}+\omega_{F S}{ }^{2}} \frac{K_{P}}{s+T_{R}}
$$

$K p$, the lateral command gain, was selected to pro$\checkmark$ ide satisfactory steady-state roll rate response. KFS was selected to provide a feel system force yradient of $4.01 \mathrm{~b} /$ in for all configurations, except one evaluation that was repeated with a 2.1) $\mathrm{lb} /$ in yradient (Configuration F).

Tasks

This preliminary investigation consisted of one flight with one evaluation pilot who was not aware of the configuration being evaluated at any yiven time. For a given configuration, precision bank-angle captures up to $30^{\circ}$ were performed on the downwind portion of the landing pattern, followed by a lateral-offset spot landing. Two approaches were typically flown in each configuration before the pilot made ratings and comment's. The evaluation flight totaled 17 approaches ( 14 to touchdown).

Results

The pilot ratings assigned to the evaluation configurations are presented in Fig. 5. Summary comments for each configuration are:

1. Configurations $A$ and $B$ (Low level of total time delay, approximately $0.15 \mathrm{sec}$ )

These configurations received pilot ratings of 2 (reyardless of the distribution of the time delay). The pilot commented on the smooth, precise control of bank angle, with no overshoot tendencies.

2. Configurations $C$ and $D$ (High level of total time delay, $0.27 \mathrm{sec}$ )

Significant differences were noted between these configurations. Configuration $C$, with the "fast" feel system, in which most of the time delay resided downstream of the feel system, received pilot ratings of 7 . A lack of precision, a tendency to overcontrol in roll and a small amplitude high-frequency lateral $P I 0$ were noted by the pilot. Configuration D, with the "slow" feel system, in which a significant portion of the total delay resided in the feel system, received pilot ratings of 4 . Some imprecision in roll was noted by the pilot, but reasonable roll attitude control was possible.
3. Configuration E (Minimal total time delay, approximately $0.10 \mathrm{sec}$ )

This "benchmark" configuration received pilot ratings of 2 , with no problems noted by the pilot.

4. Configuration $F$ (Configuration $D$ with reduced Tateral stick force gradient)

This configuration was the same as configuration $D$ except that the feel system force gradient was cut in half to 2.0 lb/in. pilot rating was a 4 , and the pilot comments indicated precise control of roll attitude and more response laterally than for the other configurations.

Discussion

The results of this limited experiment show interesting trends with respect to the MIL-F-8785C equivalent time delay requirements. The pilot ratings relative to the MIL-F-8785C requirements for the total equivalent time delay of each configuration (including the feel system) are shown in Fig. 5. Again, the degradation of flying qualities at the higher total time delay is significant when a majority of the time delay is located downstream in the transport delay and Learjet actuator, and not in the feel system. In addition, the results do not correlate satisfactorily with MIL-F-8785C requirements.

The same experiment results are shown in Fig. 6, but with the feel system time delay removed from each configuration. In this case, there is excellent correlation between pilot ratings and MIL-F-8785C boundaries, as there was for the $X-29 A$ flight test data previously discussed.

The major points brought out by this small experiment, substantiated by the flying qualities evaluations of the $X-29 A$ aircraft, are:

1. Large values of overall lateral equivalent time delay $(0.27 \mathrm{sec})$ can be satisfactorily tolerated by the pilot (pilot rating 4, Leve1 2) when a significant portion of this delay resides in the feel system, independent of the force gradient.

2. Lateral flying qualities degrade to Level 3 (pilot rating 7 ) when large values of overall time delay $(0.27 \mathrm{sec})$ consist of delay downstream of the feel system, and this delay is largely transport time delay. This effect, related to the distribution of the overall time delay, is not present at low values of overall time delay $(0.15 \mathrm{sec})$.

3. Correlation of the pilot rating results with the MIL-F-8785C time delay boundaries is poor when the feel system is included, as required by MIL-F-8785C. Excellent correlation is obtained, however, when the overall time delays 
in a position-command flight control system are referenced to stick position, not stick force, therefore excluding the feel system delay contribution.

A more yeneral comment based on data and observations presented up to this point, is that when the cockpit feel system is a significant dynamic element in the flight control system, the present MIL-F-8785C time delay requirements do not appear to be applicable. In view of the data and observations in this paper, the central point of interest is the feel system and its impact on aircraft flying qualities and related specifications.

\section{Feel System Effects on Flying Qualities}

It appears that feel system effects on flying qualities can be approached from two viewpoints. In each case the initial assumption is that the MIL-F-8785C requirements are deficient, or at least, incomplete. This assumption is consistent with previous data and observations presented in this paper.

The first point of view is a more general one in which the feel system is considered as another flight control system filter which can significantly affect the shape of the initial response of the aircraft following the time delay. Evidence in Ref. 4, for example, suggests that the allowable time delay is a function of the initial response parameters, such as roll mode time constant.

The second point of view considers the feel system to be a unique dynamic element within the fliyht control system since the pilot has direct access to both the input force and output stick displacement of the element. The pilot's ability to apply compensation directly to the feel system element as a special inner loop in the complex pilot model may possibly discount the feel system time delay contribution in some way for positioncommand flight control systems.

Feel system as a Filter

To illustrate the role of the feel system as a filter which can significantly affect the shape of the initial response of the aircraft, consider configurations $C$ and $D$ from the Calspan Learjet feel system experiment. The roll rate and roll acceleration time histories of these configurations for a step force input are presented in Fig. 7. As shown, the roll rate responses are weli matched with the same overall effective time delay. However, the roll acceleration time histories are quite different. Configuration $C$ with the fast feel system shows a delayed and abrupt initial acceleration that lead to PIO problems and a pilot rating of 7 . On the other hand, configuration $D$ with the slow, lower frequency feel system shows a slightly reduced maximum acceleration peak, but has less initial delay and a substantially reduced initial roll acceleration rate, sometimes referred to as "jerk." To the pilot, the high-frequency attenuation provided by the feel system filter was beneficial and resulted in significantly better aircraft performance (pilot rating of 4 ). With the smoothing effects of the feel system, the tolerance of time delay is increased. Whether these benefits could be obtained using appropriate filtering downstream of the feel system should be the subject of further experiments. This example does suggest that time delay tolerance is very much a function of the shape of the initial acceleration of the aircraft following the time delay dead time.

In an effort to substantiate this apparent relationship between time delay and the rate of change of acceleration or jerk, the larger data base from the LATHOS experiment of Ref. 4 was examined. This lateral flying qualities experiment showed that the allowable time delay for a given flying qualities level was a function of the roll mode time constant. For short roll mode time constants and the attendant higher initial lateral accelerations, the allowable time delay was significantly reduced.

Selected configurations from this experiment had medium-to-short values of roll mode time constant and adverse pilot comments related to rapid initial response. The acceleration rate (jerk) for these configurations was then measured for a step force input. The step input size was selected to achieve a somewhat arbitrary, but reasonable standard of roll performance; $30^{\circ}$ of bank-angle change in $1 \mathrm{sec}$ was selected. The measured values of acceleration rate and effective time delay for each selected configuration are plotted in Fig 8 . Although the data set is somewhat limited, the observations from the Learjet experiment are essentially confirmed: Allowable time delay appears to be a function of the initial response shape, in this case lateral acceleration rate. The feel system, acting as a filter, can potentially reduce the rate of acceleration which, in turn, increases the tolerance to time delay. These smoothing effects appear to more than offset the degrading effects of the additional equivalent time delay added by a lower frequency feel system filter.

This scenerio is a possible explanation for the results observed in the Learjet experiment and the $X-29 A$ flight tests. A definitive experiment to isolate the feel system effects has not yet been done. There is, however, another possible explanation for the effects of significant feel system dynamics related to the unique aspects of the feel system as a dynamic element in the flight control system.

\section{Feel System as a Unique Dynamic Element}

Typically, the feel system is treated equally with the other cascaded dynamic elements in the flight control system with respect to time delay. However, there is evidence that suggests the dynamics contributed by the cockpit feel system should be approached from a different perspective.

The feel system includes the spring, mass, and damper characteristics of the control stick that 
translate the pilot's stick force input into stick position. In a modern fliyht control system, the feel system provides the "artificial feel" necessary for the pilot to maintain adequate stick force and position feedback. In most cases, the feel system is modeled linearly by a second-order lag prefilter in the command path of the flight control system (Fig. 3). Where the flight control system is commanded by stick position (as in Fig. 3), the commanded signal directs the filight control system to provide the surface motions that result in aircraft response. Generally, the control system time delay is referenced to stick force (as required by MIL-F-8785C) which would include the feel system in a stick position command systerl.

The effect of feel system dynamics on pilot performance is illustrated using a model-based approach. Hess 10 offers a structural model for the human pilot that includes a pair of explicit proprioceptive feedback loops (Fig. 9). A significant feature of this human pilat model is the proprioceptive information, such as stick position or force from the control stick, constituting one of the major feedback paths in the model. Assuming the pilot uses stick position as his "output," the feel system dynamics can be included in the forward loop, as shown in Fig. 9. Using the slow feel system in the Learjet investigation (13 rad/sec), a root locus of the neuromuscular and feel system dynamics when the pilot closes this inner-most proprioceptive loop is shown in Fig. 10. The inner-most loop closure drives the feel system pole to higher frequencies, thus reducing the amount of equivalent time delay associated with the feel system. This model-based approach suggests that the feel system is indeed a unique dynamic element which the pilot can directly affect by his inner-most loop closures.

The previous data and observations from the $X-29 A$ and the brief Learjet feel system experiment suggest that better agreement with the MIL-F-8785C time delay boundaries could be obtained by excluding the feel system totally in a position-command flight control system. In both cases, major discrepancies were evident when significant feel system dynamics were present. Although the previous data have focused on the lateral axis, the techniques discussed in this paper should ideally apply in some form to all axes and tasks. The limited data presented in this paper are obviously insufficient to define the extent the feel system time delay should be discounted, but the observations and the analysis using the Hess model do suggest that the feel system is a unique dynamic element in the control system.

\section{Concluding Remarks}

The flying qualities problems associated with advanced electronic flight control systems often relate to the control system time delay. This paper updates the information base on the effects of time delay on flying qualities using recent flight observations from the $X-29 A$ technology demonstrator and a related preliminary flight experiment. The major points in the paper are:

1. The present MIL-F-8785C allowable time delay specifications do not appear to apply to position-command flight control systems with significant feel system dynamics. Better correlation with these time delay boundaries is obtained when the time delay measurement is referenced to stick position, not force, and the feel system is therefore excluded.

2. The allowable time delay appears to be a function of the shape of the aircraft response following the initial delay time. In particular, the limited data presented in this paper suggests that the allowable delay in the roll axis is a function of the initial acceleration rate, or "jerk."

3. There is evidence that suggests the feel system is a unique dynamic element in the control system whose delay contribution can potentially be reduced through the pilot's inner-most loop closures between stick position and stick force.

4. Flight observations indicate that the expected flying qualities degradations related to the inclusion of a low frequency, slow feel system in the control system do not materialize. In fact, the beneficial high frequency attenuation of such a feel system is obtained at little cost. This somewhat unexpected result is either because the time delay of the feel system can be largely discounted by pilot compensation or that the beneficial smoothing effects of the feel system as a filter increase the time delay tolerance.

5. A review of the present MIL-F-8785C allowable time delay specifications and the generation of new data to isolate the role of the feel system in the control system are in order. The time delay issue appears to be more complex than suggested in MIL-F-8785C and the allowable delays appear to be a function of the character and distribution of the dynamic elements in the control system.

\section{Acknowledgements}

The authors wish to express their thanks to Ronald $A$. Hess for providing insight into the potential interaction of feel system dynamics with the structural model of the human pilot.

\section{References}

1"Military Specification: Flying Qualities of Piloted Airplanes," MIL-F-8785C, 1980.

2Smith, R.E. and Bailey, R.E., "Effect of Control System Delays on Fighter Flying Qualities," AGARD Conference Proceedings No. 333 on Criteria For Handling Qualities of Military Aircraft, Apr. 1982. 
${ }^{3} A^{\prime}$ Harrah, R.C., Hodgkinson, J., and LaManna, W.J., "Are Today's Specifications Appropriate for Tomorrow's Airplanes?" AGARD Flight Mechanics Panel Symposium on Stability and Control, Sept. 1978.

4Monagan, S.J., Smith, R.E., and Bailey, R.E., "Lateral Flying Qualities of Highly Augmented Fighter Aircraft," AFWAL-TR-81-3171, Jan. 1982.

5Berry, D.T., Powers, B.G., Szalai, K.J., and Wilson, R.J., "A Summary of an In-Flight Evaluation of Control System Pure Time Delays During Landing Using the F-8 DFBW Airplane," AIAA 80-1626, Aug. 1980.

${ }^{6}$ Smith, R.E., "Effects of Control System Dynamics on Fighter Approach and Landing Longitudinal Flying Qualities," AFFDL-TR-78-122, Mar. 1978.
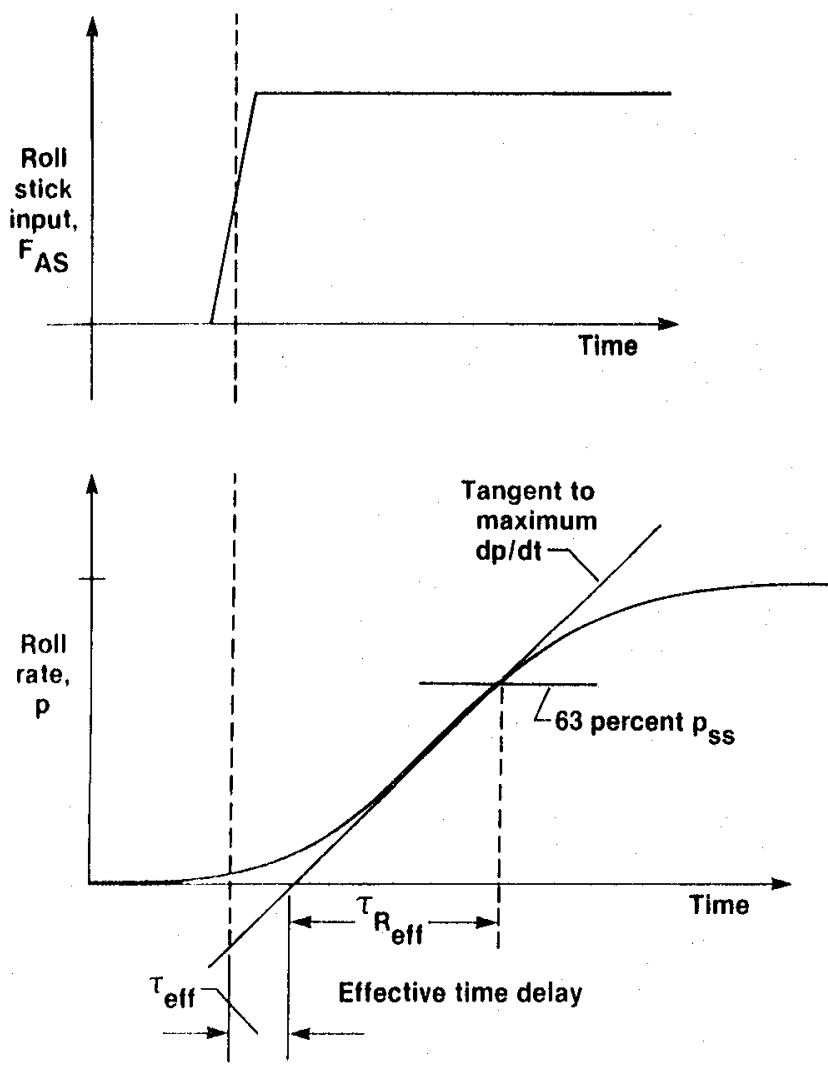

Fig. 1 Effective time delay and time constant.
7 Hodgkinson, J. and Snyder, R.C., "Flight Evaluation of Augmented Fighter Aircraft," MCAIR 80-028, Presented at the 1980 AIAA Atmospheric Flight Mechanics Conference, Aug. 1980.

8Gera, J., "Dynamics and Controls Flight Testing of the X-29A Airplane," NASA TM-86803, 1986.

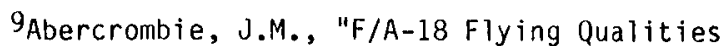
Development," MCAIR 84-009, Presented at the University of Kansas Aero Colloquium, Lawrence, Kansas, 25 Mar. 1983.

10Hess, R.A., "Structural Model of the Adaptive Human Pilot," Journal of Guidance and Control, Vol. 3, No. 5, Sept.-0ct. 1980, pp. 416-423.

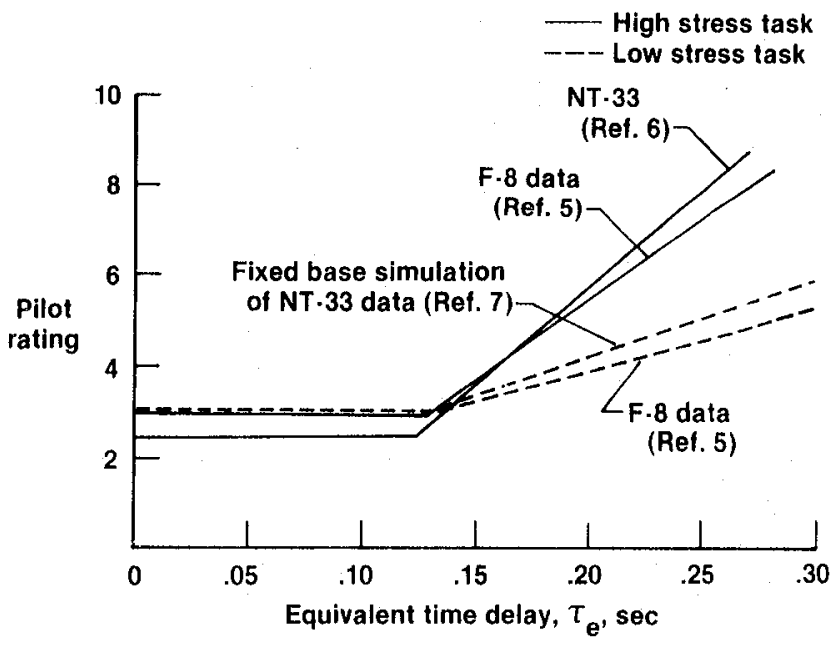

Fig. 2 Comparison of time delay effects for low and high stress landing tasks.

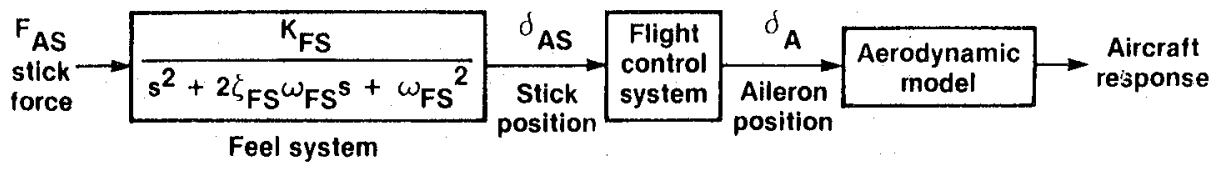

Fig. 3 Simplified lateral control system simulated in the feel system investigation. 


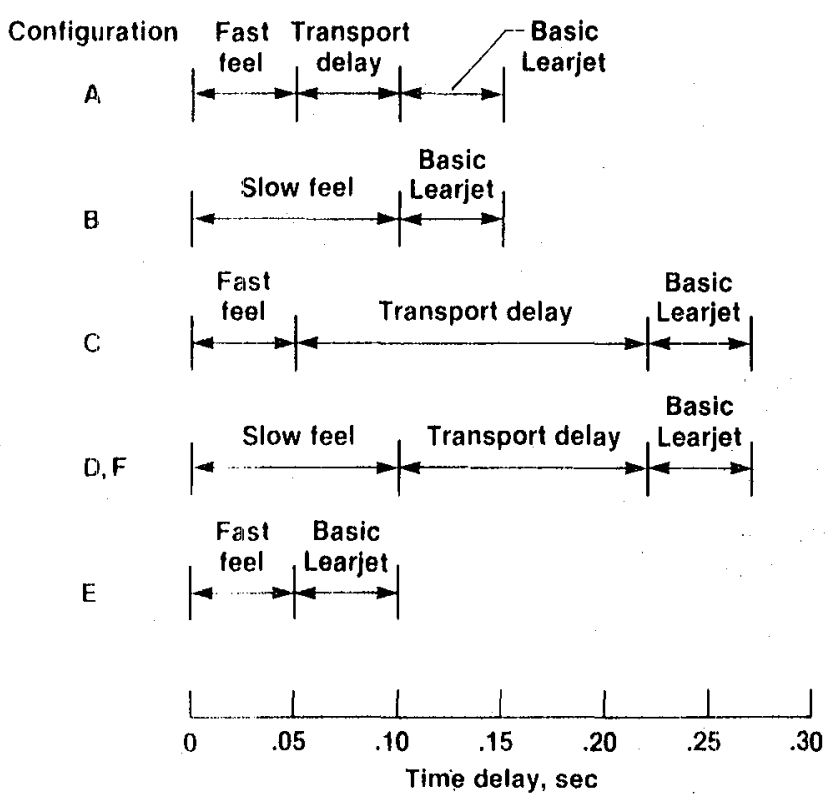

lig. 1 lateral feel system investigation, time delay structure.

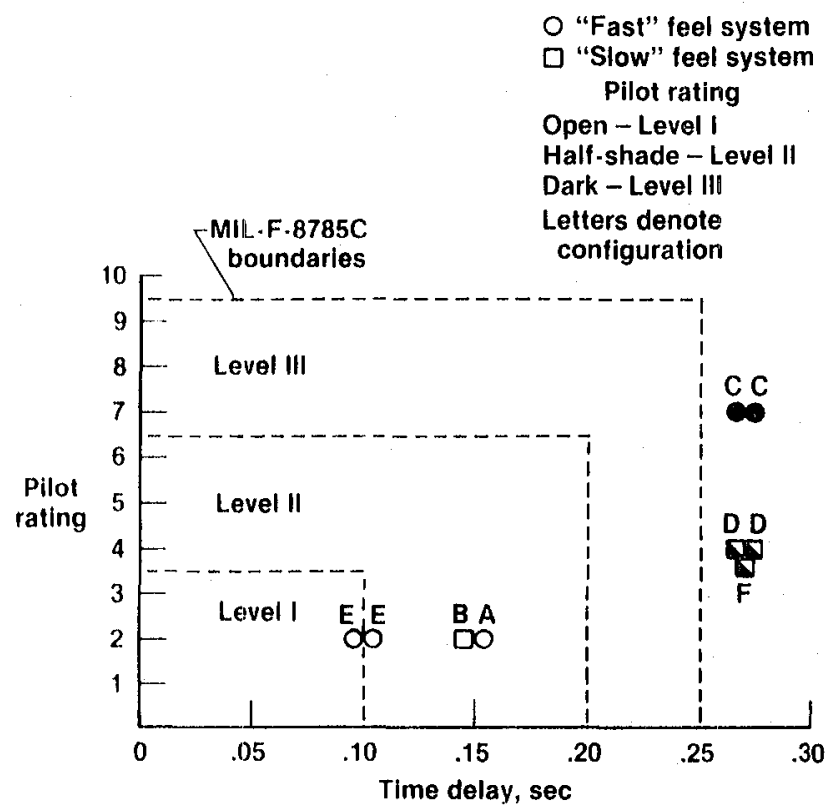

Fig. 5 Lateral feel system investigation pilot ratings (feel system included).

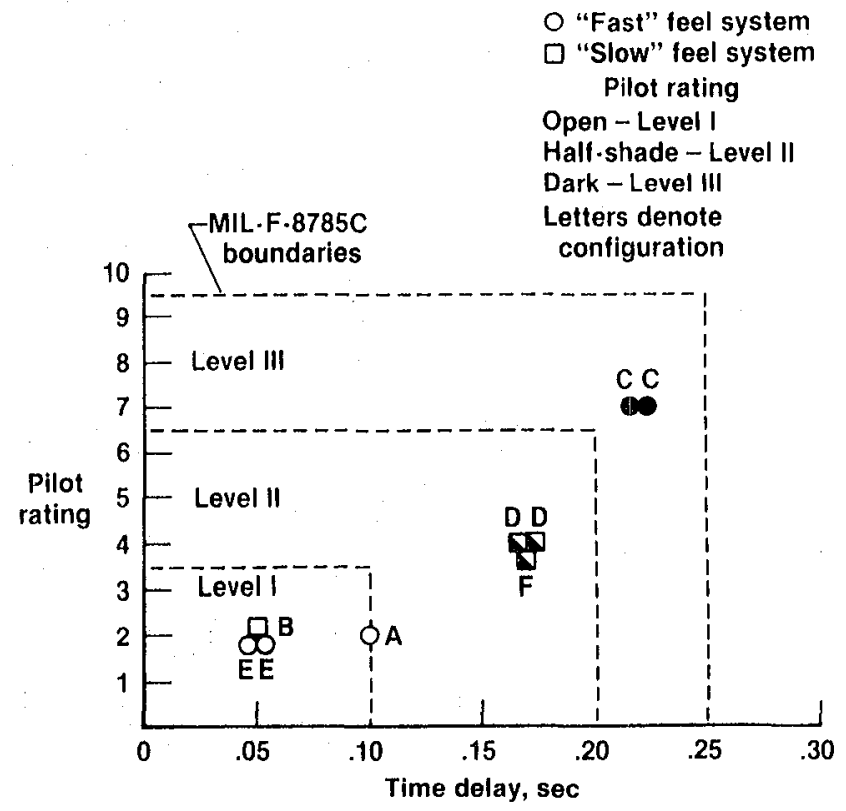

Fig. 6 Lateral feel system investigation pilot ratings (feel system excluded).

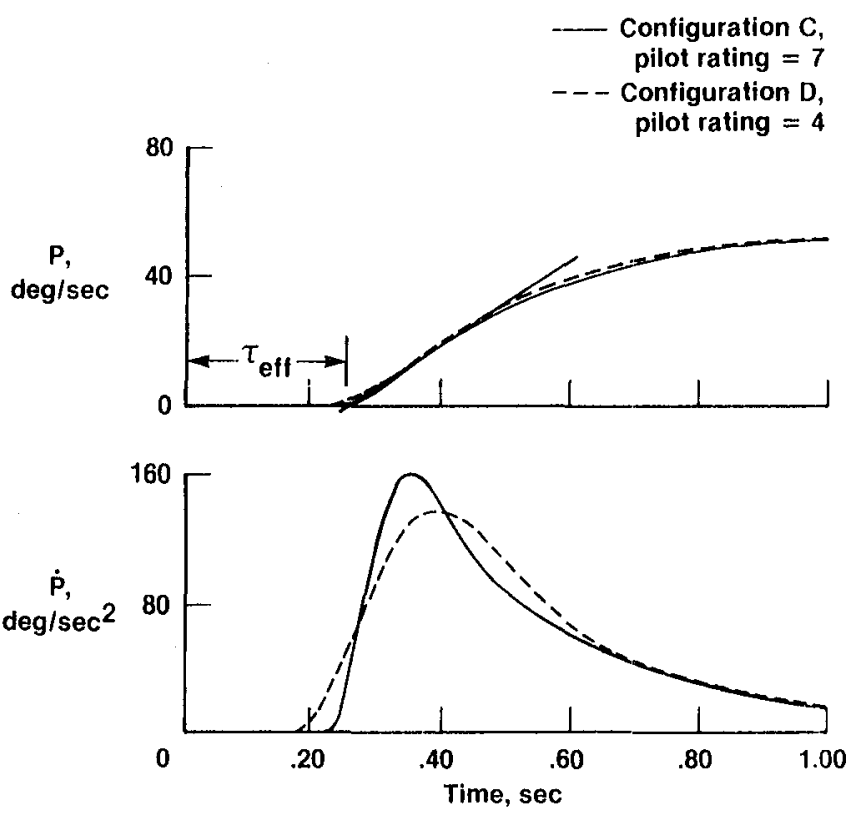

Fig. 7 Feel system effect on initial roll response (step force input). 


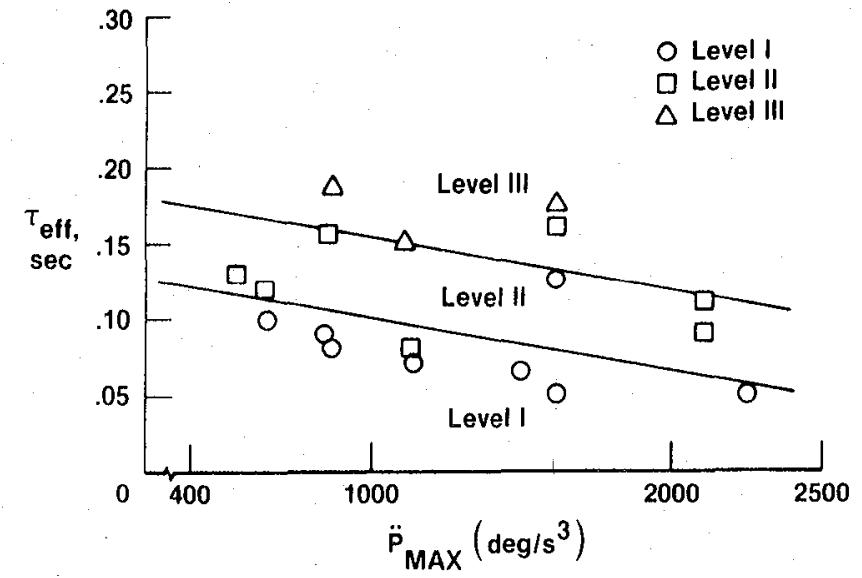

Fig. 8 Effect of acceleration rate on effective time delay (LATHOS data-category A).

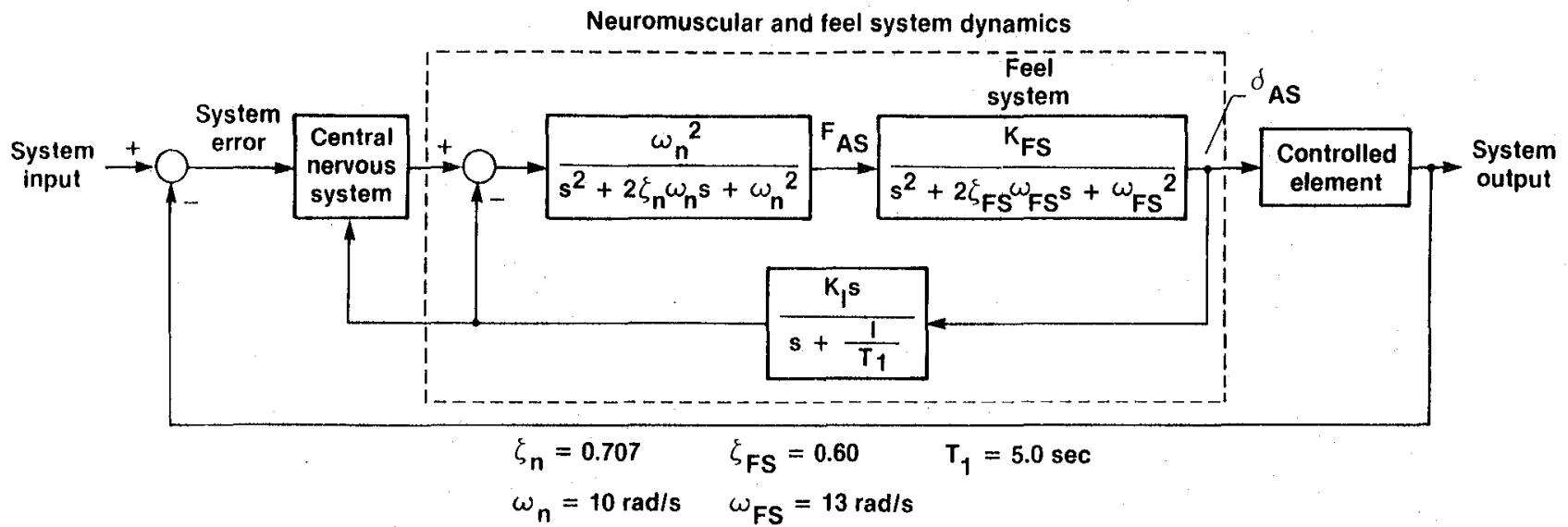

Fig. 9 Hess structural model of the human pilot.

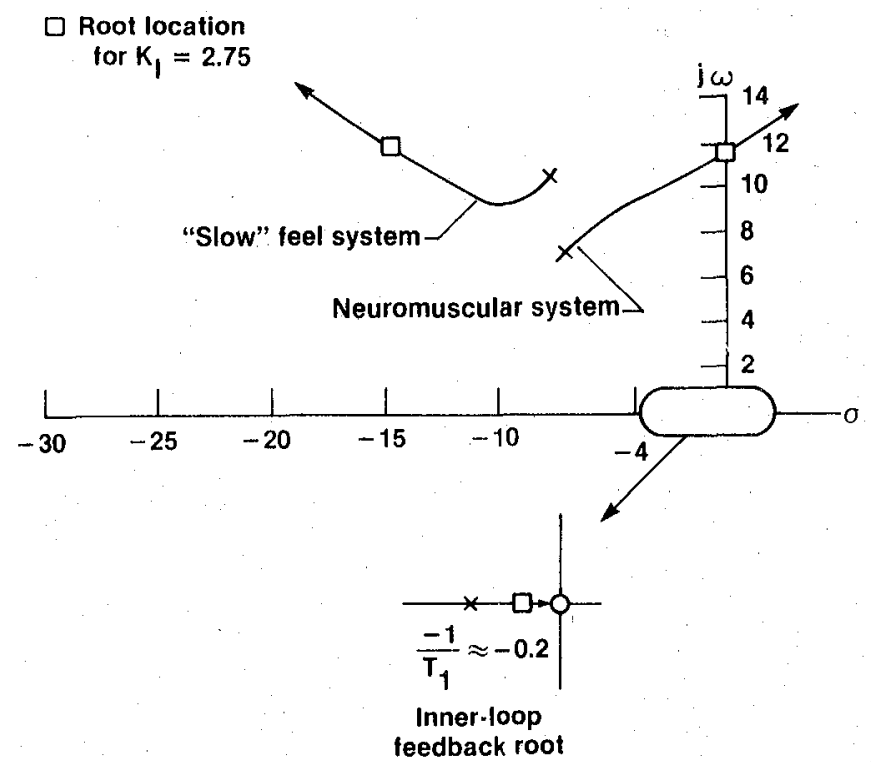

Fig. 10 Root locus of inner-most force/position Loop. 


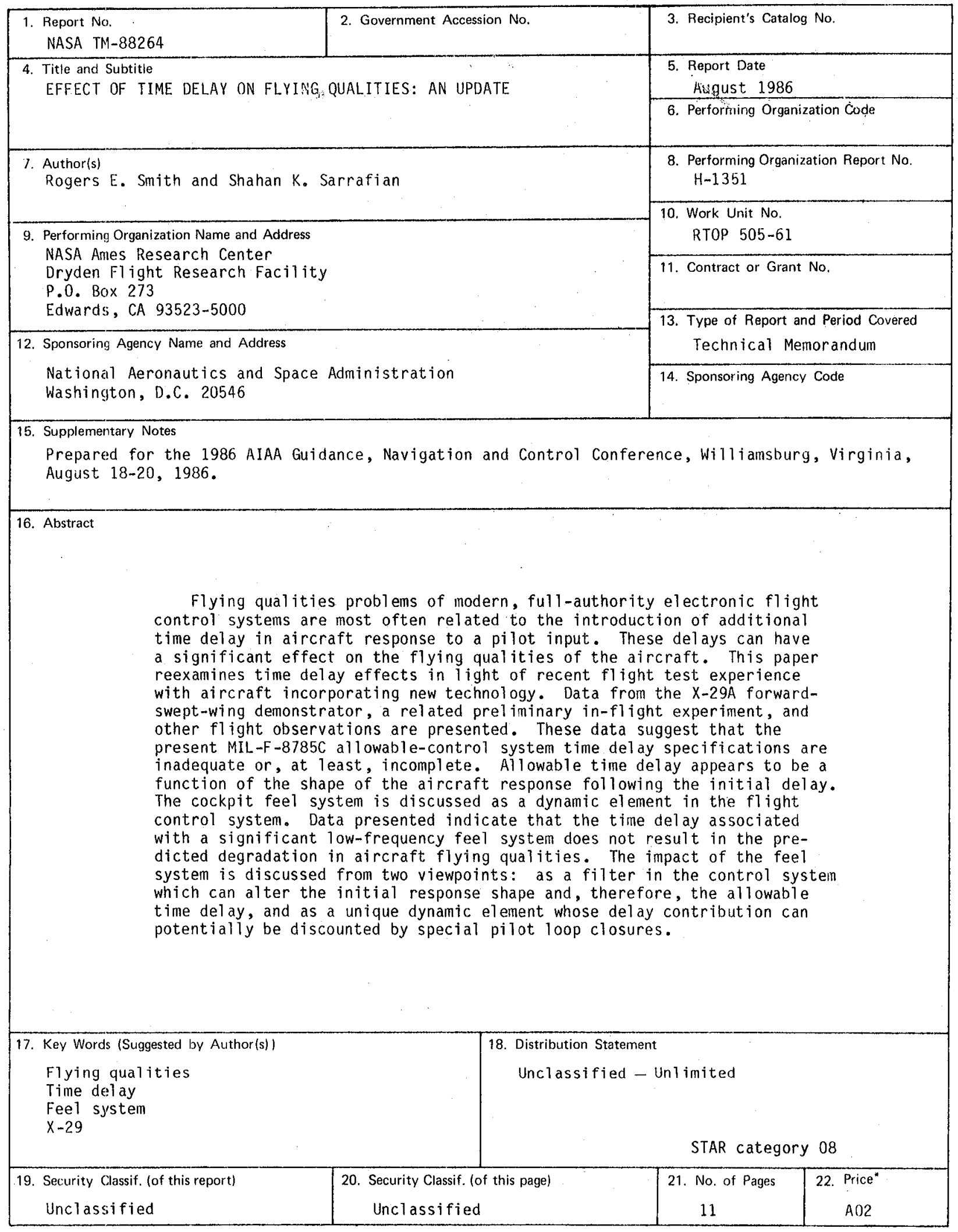

"Fox sale by the National Technical Information Service, Springfield, Virginia 22161. 
End of Document 